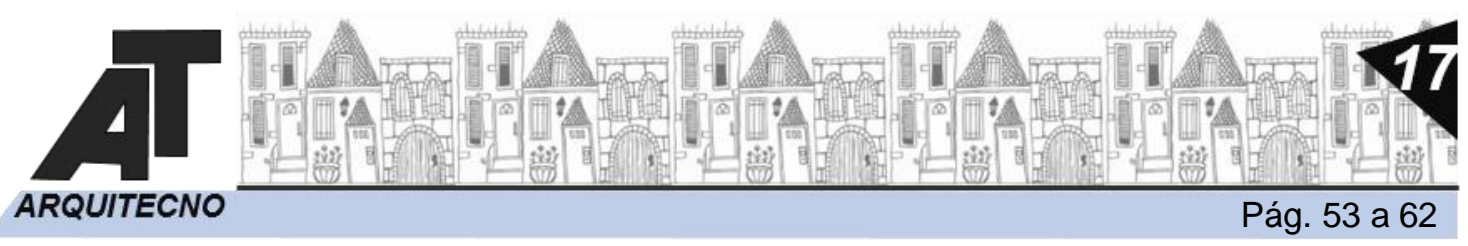

\title{
Evaluación del comportamiento térmico edilicio: un caso en el Valle Calchaquí Tucumano
}

\section{Evaluation of building thermal behavior: a case in the Calchaquí Tucumano Valley}

\author{
Amalita Fernandez, Vanesa Celina Saez, Beatriz Silvia Garzon
}

\begin{abstract}
Grupo Hábitat Sustentable y Saludable (GHabSS), Facultad de Arquitectura y Urbanismo, Universidad nacional de Tucumán (FAU-UNT), Consejo Nacional de Investigaciones Científicas yTécnicas (CONICET), Tucumán, Argentina. amalitaf93@gmail.com,vanesaez@gmail.com, bgarzon06@gmail.com
\end{abstract}

\section{RESUMEN}

La región de los Valles Calchaquíes, en Tucumán tiene un acceso limitado a las energías secundarias, esto afecta el confort térmico y dificulta establecer actividades laborables permanentes. En tal sentido, el objetivo principal del presente trabajo es mostrar cómo se puede en las etapas iniciales de diseño de un edificio definir el uso eficiente de energías naturales, mediante el uso de software de simulación grafica y analítica. Se toma como caso de estudio el anteproyecto de una fábrica de dulces en Amaicha del Valle. Se analiza la propuesta original conforme Normas IRAM. Se estudia su asoleamiento con software GEOSOL y simulación grafica (Sketch up) y su comportamiento térmico con SIMEDIF. Luego, se propone un rediseño en el cual, también, se realizan las mismas verificaciones para su comparación con el original. Se concluye que la propuesta de rediseño es la más favorable, presenta mejoras para invierno siendo la situación más desfavorable.

\section{ABSTRACT}

The Valles Calchaquíes region, in the province of Tucumán, has limited access to secondary energy sources, this affects thermal comfort and makes it difficult to establish permanent work activities. In this sense, the main objective of this work is to show how it is possible through the use of graphic and analytical simulation software in the initial stages of building design to define the efficient use of natural energies. The preliminary project for a sweetmeat factory in Amaicha del Valle, Tucumán, is taken as a study case. The original proposal is analyzed according to IRAM Standards. Its sunlight is studied with GEOSOL software and graphic simulation (Sketch up) and its thermal behavior with SIMEDIF. Then, a redesign is proposed in which, also, the same verifications are carried out for comparison with the original one. It is concluded that the redesign proposal is the most favorable, it presents improvements for winter being the most unfavorable situation.

PALABRAS CLAVES: Arquitectura solar; Confort térmico; Simulación térmica, Geometría solar.

KEY WORDS: Solar architecture; Thermal comfort; Thermal simulation, Solar geometry.

FECHA DE RECEPCIÓN: 10/2/2021 | FECHA DE ACEPTACIÓN: 12/5/2021

DOI: http://dx.doi.org/10.30972/arq.0174983 


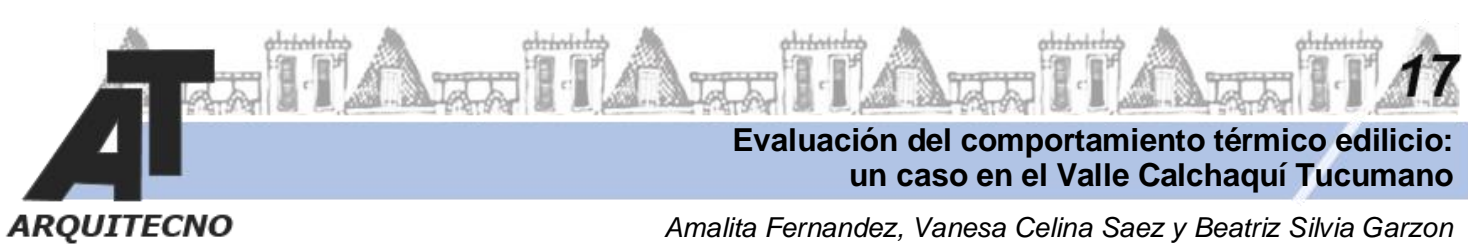

\section{INTRODUCCIÓN}

La región de las Cumbres y Valles Calchaquíes, tienen un acceso limitado a la red eléctrica y no cuenta con infraestructura para el abastecimiento de gas natural (INDEC, 2010). Esto afecta la calidad de vida de los pobladores. Por un lado, el déficit en relación con el confort térmico en el hábitat y por otro lado las dificultades de establecer actividades laborales con relación a la producción manufacturera. En tal sentido, el Instituto Nacional de Tecnología Agropecuaria (INTA,2020) expone que ante la falta de infraestructura en los Valles se origina una problemática compleja. Esta abarca distintos aspectos como, la falta de generación de empleo genuino y por ende la migración y el despoblamiento de las zonas rurales, así como también una carencia de entidades productoras consolidadas. Frente a este contexto, resulta necesario apelar a una mejor utilización de energías naturales que permita disminuir o independizar a los edificios de las energías secundarias.

El diseño arquitectónico a partir del conocimiento del clima de la región resulta indispensable para abordar un proyecto eficiente desde el punto de vista energético y que garantice el confort térmico. Por ello, se puede pensar que el diseño debe ser el campo de gestión y elaboración de los productos arquitectónicos, revalorados y reformulados por los principios del bioclimatismo en procura de edificaciones energéticamente eficientes, con una expresión formal-tecnológica acorde con su contexto (Garzón, 2007). Existen estudios sobre la aplicación de estrategias pasivas de diseño arquitectónico que demuestran una disminución considerable del consumo de energía de un edificio construido, siendo dicha reducción independiente de la fuente empleada (Fernández et al., 2020). Resulta indispensable tener un claro entendimiento de la geometría solar y su incidencia en la tierra, para poder aprovechar esta energía (Giraldo y Araldo, 2020). La incidencia de la radiación solar en edificios es un tema crítico durante la etapa de diseño, a partir de su adecuado estudio se puede aprovechar el recurso o proteger del mismo (Garzón et al., 2012), además, de mejorar el balance en la relación costo-beneficio (Kuchen y Kozak,2020). $\mathrm{Si}$ bien, el estudio en profundidad de un caso real permite establecer las oportunidades y limitaciones que operan en un contexto determinado, la modalidad de investigación a través del diseño y con la ayuda de herramientas de simulación permiten determinar situaciones que serían difícil o costosas analizar de otra manera (Kozak et al.,2017; Marino et al.,2019).

Existen investigaciones concernientes a la arquitectura bioclimática en la zona de los Valles de provincia de Tucumán. Como, por ejemplo, el estudio en relación a la aplicación de estrategias de diseño bioclimático-energético y simulación térmica en viviendas rurales de Colalao del ValleTucumán (Garzon y Giuliano Raimondi, 2008; Garzon 2010; Garzon y Mendonca, 2012). También, las relacionadas al acondicionamiento térmico en aulas escolares en Tafí del Valle, Tucumán (Ledesma et al., 2015) o las referidas al comportamiento térmico de los muros de Adobe por el Centro Regional de Investigaciones de Arquitectura de Tierra Cruda (CRIATiC, 2007). Sin embargo, se detecta que están realizadas sobre construcciones existentes. En tal sentido, el objetivo principal del presente trabajo es mostrar cómo se puede mediante el uso de software de simulación grafica y analítica en las etapas iniciales de diseño, de un edificio, definir el uso eficiente de energías naturales.

\section{METODOLOGÍA}

Se toma como caso de estudio un anteproyecto existente de una fábrica de dulces, en el campo experimental Encalilla, Amaicha del Valle, Tucumán. Esta documentación fue proporcionada por la Dirección de Alimentos de la provincia de Tucumán, en el marco del proyecto "Agregado de valor de la vegetación nativa (Productos forestales no madereros) de tierras áridas para conservar la identidad cultural de la región". Surge de las actividades convenidas dentro de una articulación realizada entre la Facultad de Arquitectura y Urbanismo de la Universidad Nacional de Tucumán (FAU-UNT) y la Dirección de Alimentos de la provincia de Tucumán perteneciente al Ministerio de Desarrollo Productivo, según Acta Acuerdo expediente № 45175/20.

Etapa 1: Análisis de las características climáticas y geográficas de Amaicha del Valle, Tucumán. Se identifica la zona bioclimática según Instituto Argentino de Normalización y Certificación IRAM 11603:2012 y sus recomendaciones de diseño. Se recopilan y examinan los datos meteorológicos actualizados de la estación meteorológica "Colalao del Valle", perteneciente a la red de estaciones de la Estación Experimental Agroindustrial Obispo Colombres (EEAOC). Por 


\section{7}

Evaluación del comportamiento térmico edilicio: un caso en el Valle Calchaquí Tucumano

Amalita Fernandez, Vanesa Celina Saez y Beatriz Silvia Garzon

ARQUITECNO

último, se determinan estrategias de diseño bioclimático por medio del diagrama de Givonni (De Casa et al., 2019) y se analiza, a partir del software de cálculo GEOSOL, la geometría solar e intensidad de la radiación para la latitud, longitud y altitud en la cual se emplaza el proyecto.

Etapa 2: Análisis térmico-energético del proyecto de arquitectura existente. El mismo se realiza a partir de una verificación del cumplimiento de las pautas de diseño para la zona bioclimática IRAM 11603:2012, de los niveles de confort higrotérmico de la envolvente opaca (Modificación №1 a la norma IRAM 11900:2017), de la calificación de sus carpinterías IRAM 11507-4:2010 y de la verificación de los coeficientes volumétricos de calefacción IRAM 11604:2001 y refrigeración IRAM 11659-2:2007. Los valores de las propiedades térmicas de los materiales industrializados involucrados se extraen de la norma IRAM 11601:2002. En tanto, para el material local utilizado, adobe, se toman los valores adoptados en el estudio de culturas constructivas en el Valle de Tucumán, presentado en 18오 SIACOT (Ortega y Garzón, 2018). En última instancia, se analiza el comportamiento solar del proyecto a partir de la simulación computacional de maquetas virtuales con el software SketchUp y se verifican los ángulos de incidencia de la radiación solar en los periodos críticos.

Etapa 3: Propuesta de rediseño y simulación térmica. En un primer momento se elabora una propuesta de rediseño de protecciones solares que contempla las estrategias y pautas de diseño bioclimáticas determinadas en la etapa 1 y la geometría solar de la localidad y del edificio determinadas en la etapa 1 y 2 respectivamente. En un segundo momento se repite el análisis de asoleamiento con SketchUp en la propuesta. Finalmente, se evalúa el comportamiento térmico de ambos proyectos a partir del software SIMEDIF para comparar los resultados y evaluar la incidencia de la mejora alcanzadas en las prestaciones térmicas.

\section{DESCRIPCIÓN DE LA SITUACIÓN}

\section{Análisis geográfico y climático de la región}

El campo experimental Encalilla, situado en Amaicha del Valle, Tafi del Valle, Tucumán según IRAM 11603, se clasifica como zona III a: templado cálido, con amplitudes térmicas superiores a los $14 \stackrel{\circ}{\circ}$. Se caracteriza por grandes rangos de temperatura durante el día, por lo cual es aconsejable el uso de todos los elementos y/o recursos que tiendan al mejoramiento de la inercia térmica. En cuanto a las orientaciones, se destaca la necesidad de proveer protecciones solares adecuadas hacia el oeste y se recomienda también que las aberturas estén provistas de sistemas que obstruyan la radiación.

A partir de la representación del clima anual, condiciones medias de cada mes (De Casa et al., 2019), se vuelcan datos obtenidos de EEAOC en el diagrama de Givonni, el cual se expresa en la figura 1. Se determina que la principal estrategia a tener en cuenta es la calefacción solar pasiva, con una incidencia del $72 \%$, seguida por la ventilación natural correspondiente al $16 \%$, y solo un $12 \%$ no necesita ninguna estrategia ya que se encuentra en la zona de confort. En cuanto a la geometría solar, la elevación y Azimut en las distintas orientaciones para el invierno y verano se expresa en la figura 2.

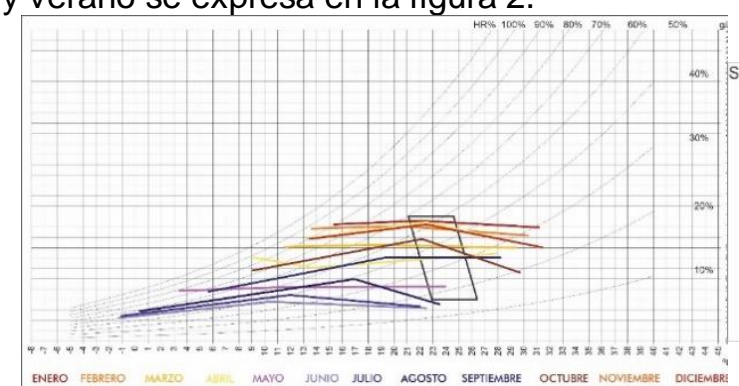

Figura 1. Diagrama psicométrico de Givonni para Amaicha del Valle. Fuente Elaboración propia a partir de De casa et al. 2019.
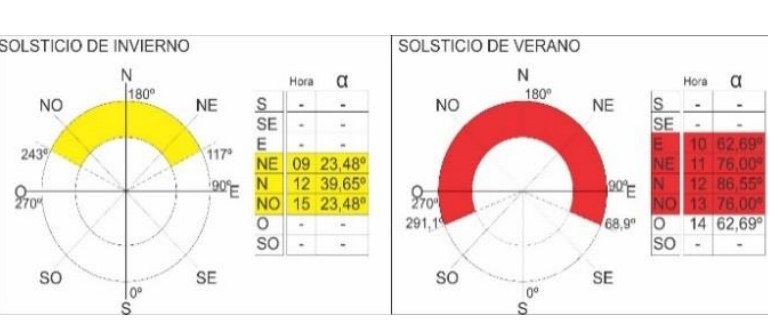

Figura 2. Geometría solar para el solsticio de invierno y verano. Fuente: elaboración propia a partir de valores obtenidos en GEOSOL.

Se observa, que la situación más crítica para esta localidad es el solsticio de invierno siendo este el día más corto del año con una duración de 10 h 19 min. La altura solar ( $\alpha$ ) máxima es de 39, $95^{\circ}$ al mediodía solar. El azimut al amanecer es de 117, es decir que la salida del sol se da hacia el noreste. Por otro lado, en el solsticio de verano, la duración del día es de 13 h 41 min, $\alpha$ 
diseño bioclimático, el proyecto original cumple con las recomendaciones generales propuestas por IRAM 11603:2012. El mismo presenta protecciones solares en las carpinterías (postigos de madera), y se materializan las paredes con adobe, material que presenta optima inercia térmica, tal como lo sugiere la norma. Desde el punto de vista térmico-energético, la envolvente se encuentra dentro de los valores recomendados por IRAM, niveles de confort higrotérmico, según su coeficiente de transmitancia térmica K (cálculo según IRAM 11601 y niveles según la modificación №1 a la norma IRAM 11900). El muro de adobe presenta un K de 1,21 W/m2K nivel $\mathrm{B}$ de confort higrotérmico para verano y $\mathrm{C}$ para el invierno; el techo presenta un coeficiente de $0,66 \mathrm{~W} / \mathrm{m} 2 \mathrm{~K}$, nivel $\mathrm{C}$ de confort higrotérmico para verano y $\mathrm{B}$ para el invierno. Las ventanas se clasifican según IRAM 11507-4:2010 como K4, con postigos cerrados, con un $\mathrm{K}$ de 2,97 W/m2K $\mathrm{y}$, con postigos abiertos, como NO clasificable con un $\mathrm{K}$ superior a $4 \mathrm{~W} / \mathrm{m} 2 \mathrm{~K}$. Se verifica el cumplimiento del coeficiente volumétrico de calefacción Gcal, expresado en IRAM 11604:2001. Este coeficiente permite evaluar el ahorro de energía de calefacción teniendo en cuenta las pérdidas de calor a través de la envolvente y por renovaciones de aire. Su valor está dado en función del volumen del local, grados días de calefacción para la localidad de estudio y nivel de aislamiento térmico de la envolvente. Se obtiene un Gcal de 1,62, siendo inferior al GRadm 2,30 lo cual implica la verificación de la norma. Por último, se calcula el coeficiente volumétrico de refrigeración (GR), valor que pondera todas las ganancias térmicas producidas en el edificio durante el verano. Los procedimientos de cálculo y verificación se encuentran en la norma IRAM 11659-2:2007. Dicho valor considera la carga térmica por radiación solar, por conducción, por ventilación y por fuentes internas. El valor obtenido es de 31,6 siendo 33,8 el admisible, a partir de ello, se verifica el cumplimiento con la norma, ya que GRadm>GR.Todos los valores obtenidos, demuestran que el proyecto original de la cocina-fábrica verifica con las normas IRAM referidas a confort térmico y eficiencia energética.

\section{Estudio de la incidencia de la radicación solar proyecto original}

Se realiza un estudio cualitativo a partir de la simulación del asoleamiento (software sketchup) en el proyecto original. Se analiza la respuesta del edificio frente al movimiento aparente del sol para los días más críticos del año: el solsticio de invierno y el de verano. Se comparan de manera gráfica las imágenes de las sombras con los valores de posición geométrica del sol obtenidos a partir del software GEOSOL para verificar su congruencia, y en consecuencia correcta implementación. En la figura 4, se observa que, durante el invierno, la galería orientada hacia el Norte genera una obstrucción total del muro y ventana Norte de la cocina, por lo cual la ganancia térmica por radiación directa resulta nula. Sin embargo, se evidencia una pequeña incidencia durante las primeras horas del día en el muro Este de la cocina. En el verano, pueden observarse incidencias en las orientaciones Este y Oeste. Pero, como las aberturas cuentan con protecciones solares móviles se puede determinar el cierre de los postigos de la ventana Oeste durante la tarde. No obstante, los postigos orientados hacia el Este no deberían cerrarse, ya que limitarían la ventilación de la cocina, por ello, sería importante contar con algún tipo de protección fija que permita mantener la ventana abierta.

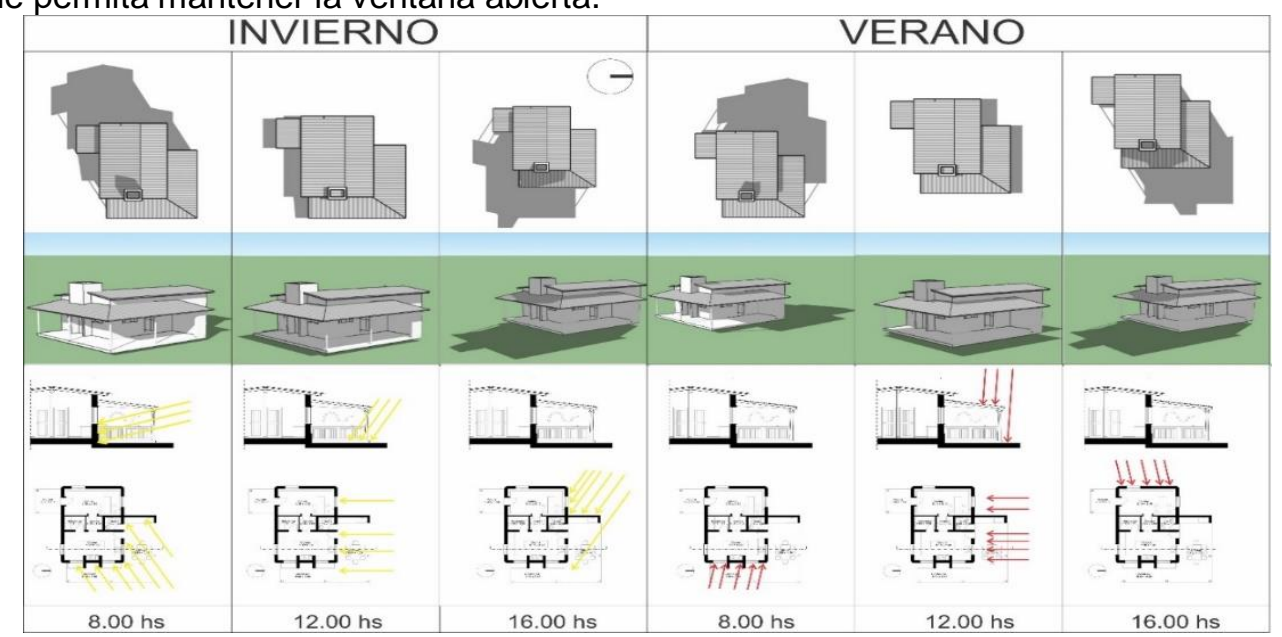

Figura 4. Asoleamiento del proyecto existente durante las estaciones críticas. Fuente: elaboración propia. 


\section{7}

Evaluación del comportamiento térmico edilicio: un caso en el Valle Calchaquí Tucumano

Amalita Fernandez, Vanesa Celina Saez y Beatriz Silvia Garzon

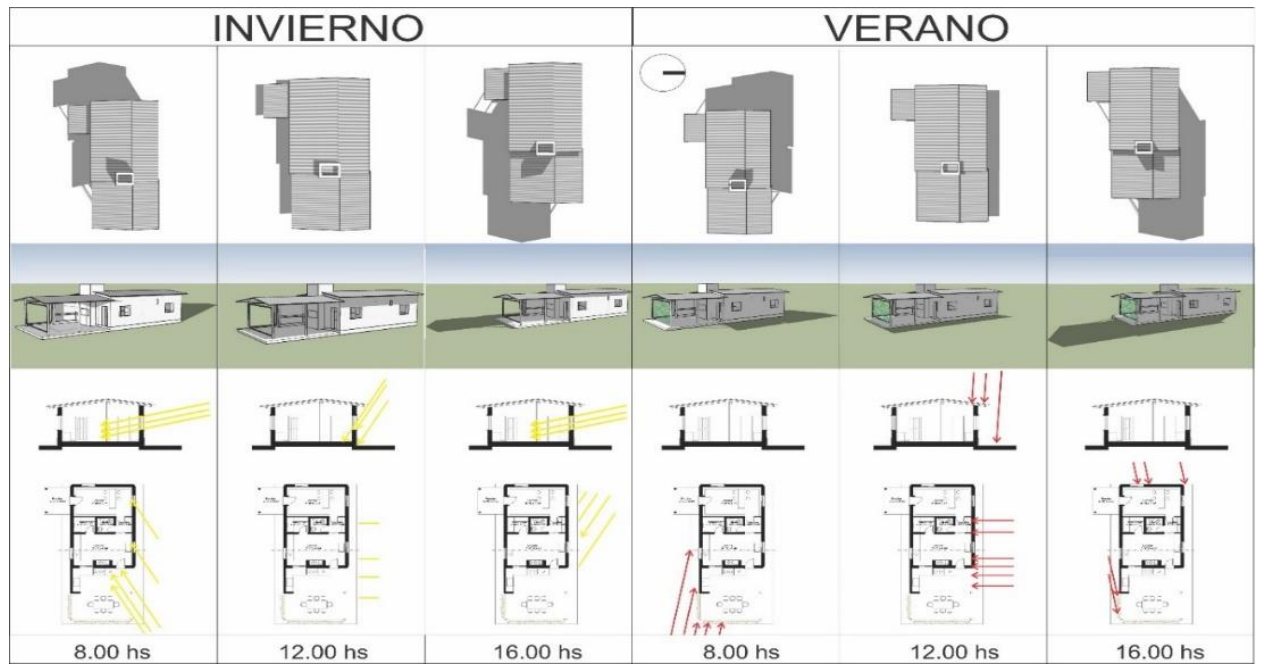

Figura 6. Asoleamiento del proyecto de rediseño durante las estaciones críticas. Fuente: elaboración propia.

Se observa la protección solar en la galería y en el área de producción y cocción en el exterior durante la estación de verano. Esto, se debe a la incorporación de vegetación de hoja caduca a modo de parasol. Asimismo, se observa la obstrucción de la radiación por el alero dispuesto al Norte. Este estudio exhibe la mejora en la respuesta del prototipo a la radiación solar.

\section{Simulación térmica}

En el presente estudio se realizan simulaciones térmicas edilicias a través del software SIMEDIF. Se cargan valores referidos al proyecto original en cuestión y al proyecto de rediseño. Las simulaciones se llevan a cabo a partir de un modelo teórico zonificado por locales: zonas térmicas. La incidencia de la radiación solar en las distintas superficies, se estiman a partir de los modelos de asoleamiento descriptos en las unidades previas. Para determinar las ganancias internas, se considera al edificio funcionando 12 horas diarias de 8 a 20 h. Se estiman cuatro personas trabajando en simultáneo. Si bien, el proyecto contempla una cocina a leña en el local de la cocina propiamente dicho, el proceso de elaboración de dulces implica tareas de preparado y envasado donde no se necesita una fuente de cocción. Se sabe, a partir de los datos climáticos analizados, que la situación más crítica es el invierno, a esta situación, se le suma la situación más desfavorable del interior artefacto cocina: apagada.

Se utilizan los datos climáticos obtenidos en la EEAOC. Dichos valores no se encuentran disponibles de manera horaria, por lo cual se estimaron a partir de los valores diarios. Esta estimación se realizó con el modelo de Parton y Logan el cual emplea una función de seno truncada para estimar las temperaturas durante el día y una función exponencial para el período nocturno (Alonso et al., 2001). Los supuestos que considera este modelo son, que la temperatura máxima ocurre en algún momento durante las horas del día antes de la puesta del sol, y que la temperatura mínima ocurre durante las primeras horas de la mañana (Parton y Logan, 1981).

En cuanto a las propiedades termo-físicas de la envolvente, se utilizaron valores normados, IRAM 11601, y los disponibles en el software de cálculo para materiales industrializados. Para los muros de adobe, se consideró una conductividad térmica de $0,46 \mathrm{~W} / \mathrm{m} 2 \mathrm{~K}$, una densidad de 1500 $\mathrm{kg} / \mathrm{m} 3$ y calor específico de $836 \mathrm{~J} / \mathrm{kg}^{\circ} \mathrm{C}$ (Ortega y Garzón, 2018). En las gráficas obtenidas en la simulación, tanto para el diseño original en la figura 7 , como para el rediseño en la figura 8 , se indica en color gris el rango de temperaturas entre $18^{\circ}$ a $26^{\circ}$, lo cual, se considera confort térmico. Para determinarlo, se toma como base los valores admisibles de temperatura operativa para espacios acondicionados naturalmente definidos por el estándar ANSI/ASHRAE 55-2004. 

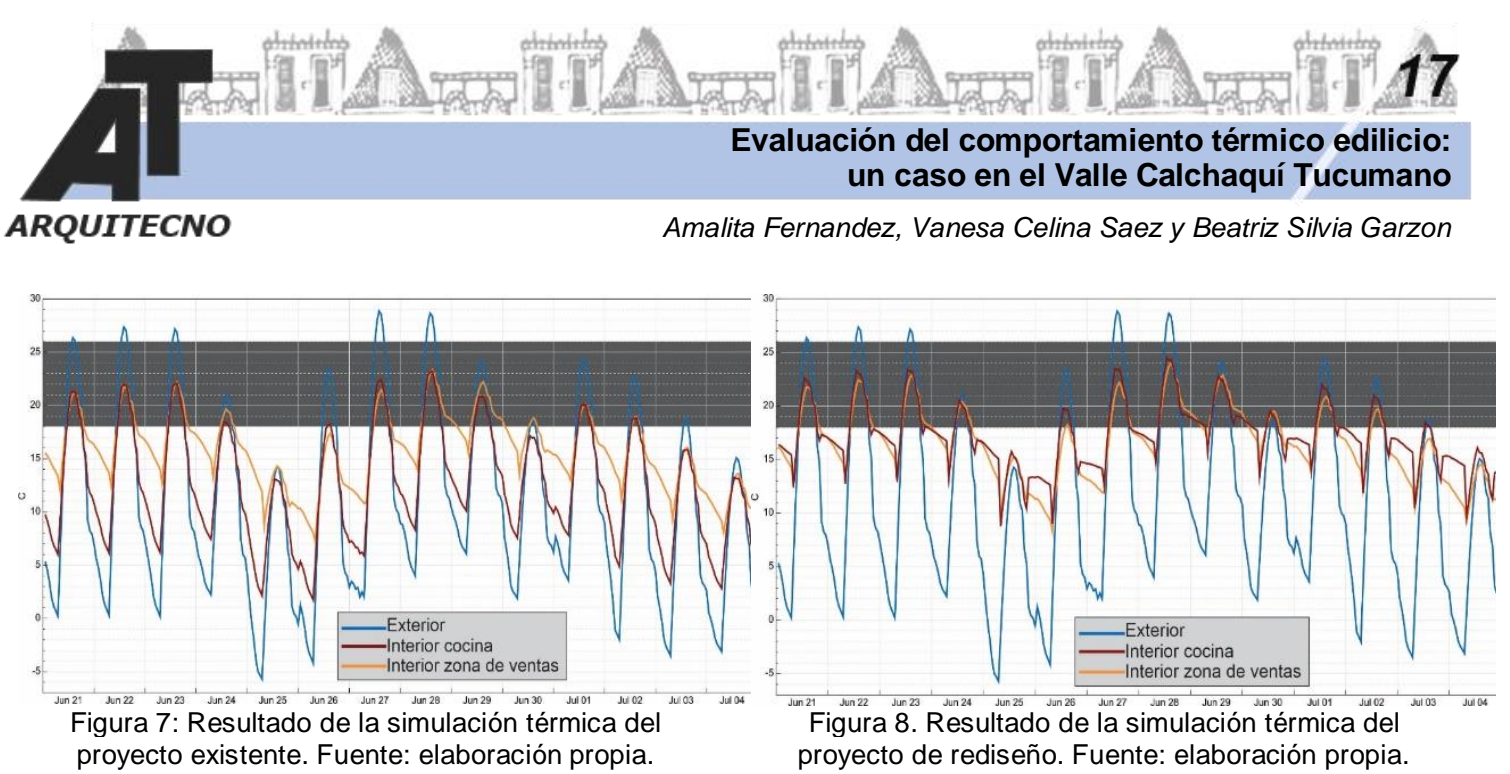

\section{Análisis de resultados}

Como se expresa en párrafos anteriores, en la zona bioclimática Illa según IRAM 11603:2012 la temperatura exterior presenta una gran amplitud que varia entre $\pm 14^{\circ} \mathrm{C}$. En referencia a esta característica, los resultados de la simulación muestran, en ambos casos, un amortiguamiento térmico en el interior del edificio. Esto se debe en gran medida a la inercia térmica que presentan los muros de la envolvente materializados con adobe. Sin embargo, existen otras variantes que considionan el retardo térmico, como lo es la ganancia solar directas por ventanas. En tal sentido, en ambas propuestas se puede observar como esta pauta de diseño, aumenta las temperaturas interiores en la situación mas desfavorable invierno. En el caso original de la figura 7, la zona térmica Interior zona de ventas presenta una menor amplitud térmica $\pm 7^{\circ} \mathrm{C}$ respecto a la zona térmica Interior Cocina $\pm 12{ }^{\circ} \mathrm{C}$. Esto se debe a que presenta una ventana orientada hacia el Norte y otra al Oeste sin ningún tipo de obstrucción, las cuales permiten la ganancia térmica durante el día. En el local cocina, si bien tiene una abertura hacia Este y otra hacia Norte, esta última se encuentra obstruida para la ganancia solar directa porque la antecede la galería. La ventaja que ofrece el mayor amortiguamiento térmico en la zona de ventas es que se necesitará menor consumo de energías naturales y/o secundarias para lograr que las 12 hs diarias de actividad permanezca dentro del rango de confort térmico. En el caso del rediseño en la figura 8, los resultados de la simulación térmica arrojan valores de temperatura interior mayores entre 1 a 1,5 ${ }^{\circ} \mathrm{C}$ para el invierno, en relación a la propuesta original. Cabe destacar, que este aumento de la temperatura interior se genera a partir de la reubicación de la galería Norte hacia la orientación Este. Esto, permite que ambas aberturas del local cocina logren ganancia térmica por radiación solar pasiva. En tal sentido, se obtiene un leve aumento en cuanto a tiempo respecto a temperaturas interiores dentro del rango de confort. Y, además, se reduce la amplitud térmica, lo que se traduce en disminución de energías naturales y/o secundarias para calefacción del recinto.

\section{DISCUSIÓN DE RESULTADOS}

En este trabajo, los resultados de la simulación sirven para comparar los valores de temperatura del proyecto original con los de rediseño, y mostrar la mejora del comportamiento térmico. Sin embargo, estos no pueden considerarse valores reales de temperatura interior, ya que no existe precisión en los datos de temperatura horaria ni se cuenta con un monitoreo térmico del proyecto existente para constatar con los valores obtenidos de la simulación, debido a que éste aún no se encuentra construido.

Por otro lado, también es importante destacar que la estrategia de calefacción solar pasiva implementada "ganancia directa por ventanas" genera un incremento de la temperatura interior, pero es insuficiente para alcanzar temperaturas de confort de manera constante durante las 12 hs de actividad de personas en la fábrica. Se abre la discusión sobre la necesidad de adoptar otras estrategias de ganancia térmica pasiva como ser un muro Trombe y verificar si, su implementación, permite alcanzar temperaturas de confort. Por último, es necesario resaltar que el estudio realizado no analiza ni cuantifica el ahorro energético, dado que el proyecto original no cuenta con ninguna estrategia activa de calefacción. 


\section{CONCLUSIONES}

A partir de la verificación de los resultados presentados, se concluye que las estrategias de diseño utilizadas en el proyecto original de la cocina-fábrica son adecuadas según el análisis normativo IRAM. Sin embargo, con base en los resultados del estudio de trayectorias solares (software Sketchup y GEOSOL) y posterior simulación térmica computacional (software SIMEDIF), se determina que la correcta disposición de las galerías mejora el comportamiento térmico del edificio analizado. De este modo. Se disminuye el consumo de energías naturales y/o secundarias para su acondicionamiento térmico. Asimismo, cabe destacar la importancia de estudiar las protecciones solares desde las etapas iniciales para garantizar un mejor comportamiento térmico, incluso cuando se trate de un edificio orientado de manera adecuada. Una disposición errónea de las mismas puede obstruir ganancias por radiación aprovechables y/o permitir el ingreso durante la estación estival, lo cual incide en la temperatura de los espacios interiores.

Un aporte significativo del presente trabajo se basa en que la intervención realizada no implica ningún tipo de costo extra para su materialización. Esta afirmación, se basa en que se emplean los mismos materiales, se conserva la superficie de la galería original, con distintas proporciones, y se propone el mismo tipo de técnica constructiva. Se destaca la utilidad de disponer de herramientas informáticas que permitan analizar el proyecto de un edificio, plantear las estrategias de diseños más convenientes desde las primeras instancias de proyecto, lo que descarta una posterior rehabilitación energética del edificio y todos los costos que esto implica.

\section{BIBLIOGRAFÍA}

Alonso, M. R., Rodríguez, R. O., \& Gómez, S. G. (2001). La utilización de las temperaturas máxima y mínima diarias para la estimación de la disponibilidad de horas de frío. Revista de la Facultad de Agronomía, 21(3), 261. http://ri.agro.uba.ar/files/download/revista/facultadagronomia/2001alonsomr.pdf

ANSI/ASHRAE (2004). Standard 55-2004. Thermal environmental conditions for human occupancy, 744.

Centro Regional de Investigaciones de Arquitectura de Tierra Cruda (CRIATiC, 2007). Comportamiento térmıco de muros de tıerra en Tucumán, Argentına. Disponible en $<$ http://fci.uib.es/digitalAssets/177/177906_4.pdf>. Fecha de acceso en 22 de octubre 2020.

Da Casa Martín, Fernando, Celis D’amico, Flavio, \& Echeverría Valiente, Ernesto. (2019). Metodología para elaborar una cartografía regional y aplicar estrategias bioclimáticas según la carta de Givoni. Revista hábitat sustentable, 9(2), 5263. https://dx.doi.org/10.22320/07190700.2019.09.02.05

Fernandez, A., Garzón, B., \& Elsinger, D. (2020). Incidencia de las estrategias pasivas de diseño arquitectónico en la etiqueta de eficiencia energética en Argentina. Hábitat Sustentable, 10(1), 56 - 67. https://doi.org/10.22320/07190700.2020.10.01.05

Garzón, B. (2007). Arquitectura Bioclimática, Nobuko.

Grazon, B. (2010). Soportes bioambientales aplicados para el diseño y construcción cogestionarios de viviendas sociales en Colalao del Valle, Tucumán. Avances en Energías Renovables y Medio Ambiente 14, pp. 05.19-25

Garzon, B. y Giuliano Raimondi, G. (2008). Aplicación de estrategias de diseño bioclimaticoenergético y simulación térmica en vivienda rural de Colalao del Valle, Tucumán como soportes para su mejoramiento. Avances en Energías Renovables y Medio Ambiente 12, pp.05.89-96. Impreso en la Argentina. ISSN 0329-5184

Garzón, B. y Mendonca, C. (2012). Monitoreo y simulación térmica de dos viviendas sociales unifamiliares bajo condiciones reales de uso en la localidad de Colalao del Valle, Tucumán. Avances en Energías Renovables y Medio Ambiente 16, pp. 05.39-44. 
